\title{
Autonomy and decentralized decision making in logistics: new ideas, technological innovations, enhanced decision support, and initial applications
}

\author{
Herbert Kopfer · Jörn Schönberger
}

Published online: 2 April 2011

(C) Springer-Verlag 2011

The last decade has revealed a profound paradigm change with respect to the organization and control of logistic systems. Forced by recent trends in the organization of enterprises and new market requirements, logistic systems are confronted with new prospects and challenges that do not fit with the paradigm of central planning. The involvement of several decision making units in one supply chain, the management of real-time data, the division of work and decision making, and a high market dynamic require innovative decision support and business information concepts. Additionally, wide-area-computer networks, ubiquitous computing, and 24-h-data availability provide a data basis as well as an infrastructure for a joint decision making among autonomous entities (e.g. agents). While the paradigm of a monolithic central control of all activities has been in the focus of research and application for several decades, the last years have revealed the intrusion of noncentralized approaches for designing, configuring, and deploying complex systems.

More than in other disciplines, there is a paradigm shift in logistics from hierarchical systems to heterachical systems, especially concerning the design and control of compound systems. In order to explore and establish a base for using and exploring the capabilities of distributed decision making, fundamental research must be executed. In the Collaborative Research Centre 637 "Autonomous Cooperating Logistic Processes-A Paradigm Shift and its Limitations", funded by the German Research Foundation, an interdisciplinary group of scientists investigates the

H. Kopfer $(\bowtie) \cdot$ J. Schönberger

Lehrstuhl für Logistik, FB 7, Universität Bremen,

Wilhelm-Herbst-Straße 5, 28359 Bremen, Germany

e-mail: kopfer@uni-bremen.de prospects and limitation of the interactive decision making among several components of a logistic system.

The components of a hierarchically organized logistic system are externally controlled units, which have only limited decision rights. On the other hand, in heterarchical systems, the components constituting a compound system are autonomous units, which interact with each other on their own responsibility, and they are provided with local intelligence and emancipated decision authority. Heterarchical structures grant autonomy to the single system components in order to enable decentralized decision making. Autonomy of components presupposes that interactive units in non-deterministic systems are able to decide and act on their own authority.

Autonomous units representing components of a complex logistic system can be found on different levels of appearance and in several contexts. At the lowest level, there are agents representing autonomous physical logistic units like parcels or containers, which are capable and allowed to decide on their handling. At the medium level, there are autonomous planning agents like human schedulers or software agents being responsible for the decisions in a delimited problem area and cooperating with agents responsible for adjacent areas. Finally, at the upper level, there are autonomous organizational units, e.g., profit centers of an enterprise or partners in a collaborative system constituting a coalition following at least one common goal.

In practice, most complex logistics systems are built in a hierarchical manner. Currently, there is a tendency to redesign such systems in a heterarchical way by constituting a set of interrelated, partly autonomous, components for the construction of the total system. The objective of the redesign is to achieve a higher degree of robustness and a positive emergence of the total system [1] by increasing 
the autonomy in the logistic systems and, simultaneously, making the complexity of a global system more tractable. Recent research on complex logistics systems aims to explore the risks and benefits of autonomy of logistical (sub-) systems and tries to develop techniques for enabling autonomy [2] for typical, formerly hierarchical, logistics systems. Additionally, techniques for determining and installing a suitable or even optimal degree of autonomy for complex logistic systems are investigated [3]. According to this trend, the tasks of solving the involved planning problems of autonomous components of a compound system are assigned to individual planning agents. These agents are not acting independently, because the coupling of the resulting subproblems requires that the relevant impacts between them will be reflected. In homogeneous situations, the coupling refers to a set of related subproblems with similar structure. Thus, all planning agents apply in principle the same solution process for the problem assigned to them. However, the solution process of an agent can be individually adapted to general global (external) requirements and to its internal local requirements. The aggregation of all subproblems constitutes a superior decision problem that corresponds to the total problem of the original complex logistics system. The superior problem may have relevant constraints and goals of its own. These superior constraints and goals cannot be judged or even be met by a single subproblem but only by the concerted behavior of the agents of all subproblems working together in a collaborative way. The relationship between the single subordinated problems and the relationship of each subordinated problem to the total superior problem must be respected during the solution process by adaptations made by the involved agents. By means of a coordinated strategy, the autonomous planning agents achieve the simultaneous and harmonized consideration of subordinated and superior requirements.

There are two typical situations for homogeneous sets of subproblems forming a superior problem. In the first situation, the subproblems are autonomous nodes that are combined by a spatial (or logical) relation to a superior problem. In this case, the superior problem is given by a resulting network of cooperating local partners. In the second case, the subproblems are combined by a temporal relation inducing a sequence in which the subproblems must be solved. The sequence of short-term problems is considered, and their combination to a superior problem yields the corresponding long-term problem. Each temporal subproblem tries to achieve its own short-term goals and concurrently has to beware the long-term goals, which can only be reached by the entirety of the subproblems. Thus, the coordination process between the subproblems and their superior problem refers to the adjustment of short-term and long-term planning.

The left part of Fig. 1 shows a spatial relation between several partners constituting the components of a superior system. Each of the partners respects their individual optimization problem with their own goals and constraints. Each of them acts autonomously, and all of them cooperate with each other. The entire system has to strive for global goals and to respect global constraints. These goals and constraints are essential for each single partner in order to survive on the long run. Sometimes the global goals of the entire coalition conflict with the individual goals of the partners. Then, the goals of the partners must conjointly be adapted to the overall requirements of the entire coalition.

Figure 1 (right picture) shows a temporal relation combining several short-term problems to a long-term problem. This is the situation investigated in the context of online optimization. The online planning process consists in a sequence of problem instances generated by updating the problem situation. The optimization of a single instance is performed on the basis of the current situation resulting from the so far pursued planning process and the newly incoming data. Each instance of the online planning problem pursues its short-term goal using its currently available data. Consequently, the planning process of each
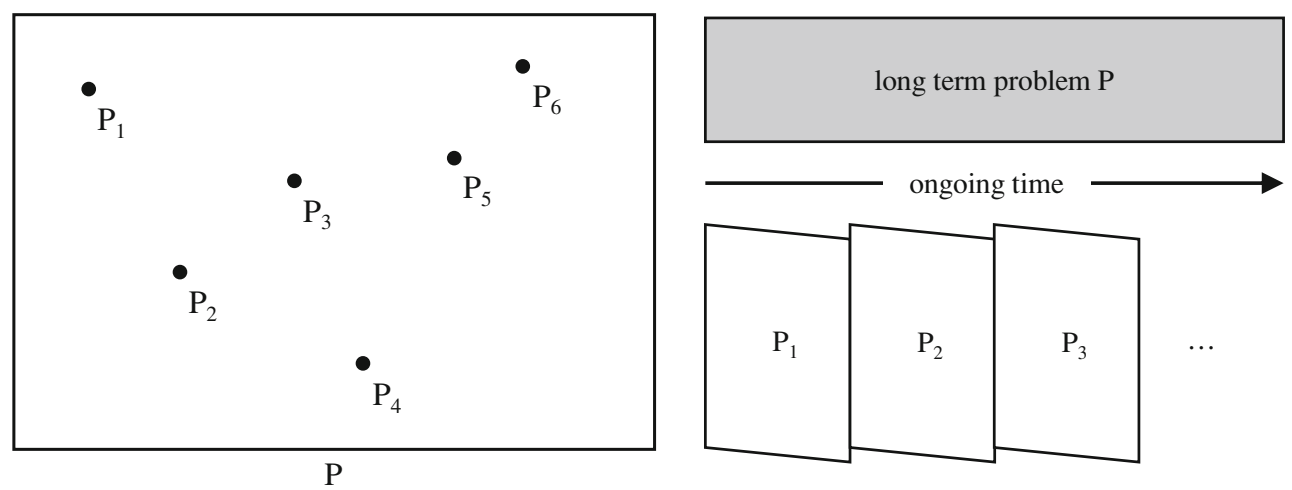

Fig. 1 Spatially related optimization problems $P_{i}$ (belonging to the partner $i$,) of the optimization problem $P$ of the entire coalition (left picture). Temporal relation of subproblems $P_{1}, P_{2}, \ldots$ (right) 
single instance (short-term problem) generates short-term solutions. Taking the actual solutions of all short-term problems together, they constitute a long-range solution for the entire long-term problem. This long-range solution is given by the concatenation of all generated short-term solutions. However, the original long-term problem to be solved refers to the entire online optimization problem for the whole planning period. It may have its own long-term goals that are to be achieved by the optimization process. Of course, the concatenation of the short-term solutions does not necessarily comply with the predefined long-term goals. Thus, each instance of the online optimization problem has to be adjusted to both, to the actually incoming new problem data and to the actual state of reaching the predefined long-term goals.

The sequence of consecutively solved instances of an online optimization problem resembles a network of problem instances where each single instance represents a node (partner) with its subordinated goal. The short-term objective of a single online problem instance corresponds to the subordinated goal of a single node, and the superior goal of the network consists in pursuing the long-term strategy for the entire planning interval. Since the concatenation of the short-term objectives of all single instances sometimes contradicts the superior goal, a model correction might be necessary from time to time. The superior goal is invisible for a single instance and can only be reached by the entirety of all nodes. Using model definition rules, it is possible to harmonize the short-term goals of single problem instances with the long-term goal of the collectivity of all problem instances over a longer time period.

Figure 1 may be useful for showing a generalized view on the relations between autonomous systems. Approaches, methods, and scenarios for autonomous systems are currently investigated in many logistical applications in various scenarios and application domains, and general research on the autonomy has attracted a lot of attention in logistics. In issues 3-4 (2010) of this journal, there have already been three contributions on this challenging subject. To push the compilation of research results related to this challenging topic, we have called for contributions to a special issue on "Autonomy and Decentralized Decision Making in Logistics: New Ideas, Technological Innovations, Enhanced Decision Support and Initial Applications." We have received 19 submissions that have been sent out to at least two reviewers each. Finally, nine papers were qualified for a publication in logistics research. In issue 1 (2011), two of these contributions have been published. Libert and ten Hompel focus on the growing complexity of modern material handling systems. Their paper introduces an ontologybased model for the description of information needed for communication among software agents in distributed material flow systems. The contribution by Jeon, Kim, and
Kopfer refers to the routing of automated guided vehicles, which autonomously compete for paths within an automated container terminal. It suggests a method for the determination of shortest-time routes by coordinating the routes of competing vehicles on the basis of the estimation of the waiting times that result from the interferences among the vehicles during traveling.

This current Special Issue of Logistics Research contains seven additional original contributions on autonomy and decentralized decision making in logistics. The paper by Montreuil suggests exploiting the decentralized features and the inherent autonomy of the Digital Internet metaphor to develop a Physical Internet vision toward meeting the grand challenges for modern logistics, especially with respect to sustainability. Zöbeley, Minner, and Kilger present and validate a management game that focuses on the conflicts occurring at the manufacturing-marketing interface. They discuss how individual behavior under distributed decision making can be improved to comply with overall company objectives. The results of their empirical investigation show that an aligned bonus scheme as well as information and communication increase the overall performance and decrease frictions between the two functional areas. The paper of Dai and Chen is about carrier collaboration in transportation. In their paper, they propose a multiagent and auction-based framework, which is implemented by a decentralized, asynchronous, and dynamic approach for transportation request exchange among carriers belonging to a given coalition. Berger and Schröder present a dynamic programming approach for collaborative forwarding of air cargo freight. Their approach enables decision makers to align their decisions in decentralized situations with the decisions of their partners commonly involved in the complex transportation chain while keeping the sensitive information of partners local and sharing only the most necessary information. The paper of Ansola, Higuera, and Otamendi focuses on the distributed decision making in airport ground management. It introduces an agent-based approach for managing handling operations supported by feedback coming from Radio frequency identification systems. The used strategies are based on a distributed organization model enforcing the division between physical elements and information technology. The contribution by Dahkovskiy, Görges, Kosmykov,Mironchenko, and Naujok presents methods and tools for modeling autonomously controlled production networks. The presented methods and tools are used for the investigation into stability properties and for the derivation of sufficient conditions for the stability of a network with stable subsystems. Finally, in the last original work presented in this Special Issue, Kreowski, Kuske, and Totth apply communities of autonomous units for the modeling of production networks with discrete processes. The 
framework for modeling distributed production networks introduced by them is founded on rule-based graph transformation and allows specifying autonomous units in such a way that they can run in parallel and can make their decisions about future actions independently of each other.

The realization of this special issue was supported by several persons. We thank you very much for their contributions. An international team of more than 25 colleagues has timely provided qualified reviews. Their expertise was invaluable for ensuring the high scientific quality of this special issue. The editorial board of Logistics Research as well as the Springer-Verlag supported our idea to dedicate a complete issue to decentralized decision making in logistics. Finally, we would like to mention the support by the German Research Foundation (DFG) as part of the Collaborative Research Centre 637 "Autonomous Cooperating Logistic Processes" (Working Group Scenarios/Modeling/Methods).
We hope you enjoy reading this Special issue of Logistics Research.

Herbert Kopfer,

Jörn Schönberger,

Editors of this Special Issue of Logistics Research

March 2011

\section{References}

1. Windt K, Hülsmann M (2007) Changing paradigms in logistics: understanding the shift from conventional control to autonomous cooperation and control. In: Hülsmann $\mathrm{M}$, Windt $\mathrm{K}$ (eds) Understanding autonomous cooperation and control in logistics. Springer, Berlin, pp 1-22

2. Hülsmann M, Windt K (eds) (2007) Understanding autonomous cooperation and control in logistics. Springer, Berlin

3. Hülsmann M, Scholz-Reiter B, Windt K (eds) (2011) Autonomous cooperation and control in logistics. Springer, Berlin 